\title{
ASYMPTOTIC FORMULA FOR THE MOMENTS OF MINKOWSKI QUESTION MARK FUNCTION IN THE INTERVAL $[0,1]$.
}

\author{
GIEDRIUS ALKAUSKAS
}

\begin{abstract}
In this paper we prove the asymptotic formula for the moments of Minkowski question mark function, which describes the distribution of rationals in the Farey tree. The main idea is to demonstrate that certain a variation of a Laplace method is applicable in this problem, hence the task reduces to a number of technical calculations.
\end{abstract}

Keywords: Minkowski question mark function, Laplace method of asymptotic expansion, Farey tree, moments of distribution

Mathematics subject classification: 11A55, 26A30, 41A60.

\section{INTRODUCTION AND MAIN RESULT}

The Minkowski question mark function ?(x) is defined for real numbers in the interval $[0,1]$ via the expression

$$
?\left(\left[a_{0}, a_{1}, a_{2}, a_{3}, \ldots\right]\right)=2\left(1-2^{-a_{0}}+2^{-\left(a_{0}+a_{1}\right)}-2^{-\left(a_{0}+a_{1}+a_{2}\right)}+\ldots\right),
$$

where $x=\left[a_{0}, a_{1}, a_{2}, a_{3}, \ldots\right]$ stands for the representation of $x$ by a (regular) continued fraction [7] (note that for $x \in[0,1)$ we have $a_{0}=0$ ). This function was proved to be continuous, monotonic and singular [5]. For convenience we introduce the Minkowski question mark function $F(x)$ on the whole non-negative real line by

$$
F\left(\left[a_{0}, a_{1}, a_{2}, a_{3}, \ldots\right]\right)=1-2^{-a_{0}}+2^{-\left(a_{0}+a_{1}\right)}-2^{-\left(a_{0}+a_{1}+a_{2}\right)}+\ldots
$$

Thus, $2 F(x)=$ ? $(x)$ for $x \in[0,1]$. The function $F(x)$ as a distribution function is uniquely determined by the functional equation [1]

$$
2 F(x)=\left\{\begin{array}{cll}
F(x-1)+1 & \text { if } \quad x \geq 1 \\
F\left(\frac{x}{1-x}\right) & \text { if } \quad 0 \leq x<1 .
\end{array}\right.
$$

This implies $F(x)+F(1 / x)=1$. The number-theoretic significance of the function $F(x)$ is apparent from the fact that it is exactly the limit probabilistic distribution of rationals in the $n$th generation of the Calkin-Wilf tree [4] (which is just a permutation of the SternBrocot tree; the Farey tree is a subtree of both). This tree is defined as follows. Starting from the root $\frac{1}{1}$, each fraction generates two offsprings

$$
\frac{a}{b} \mapsto \frac{a}{a+b}, \quad \frac{a+b}{b}
$$




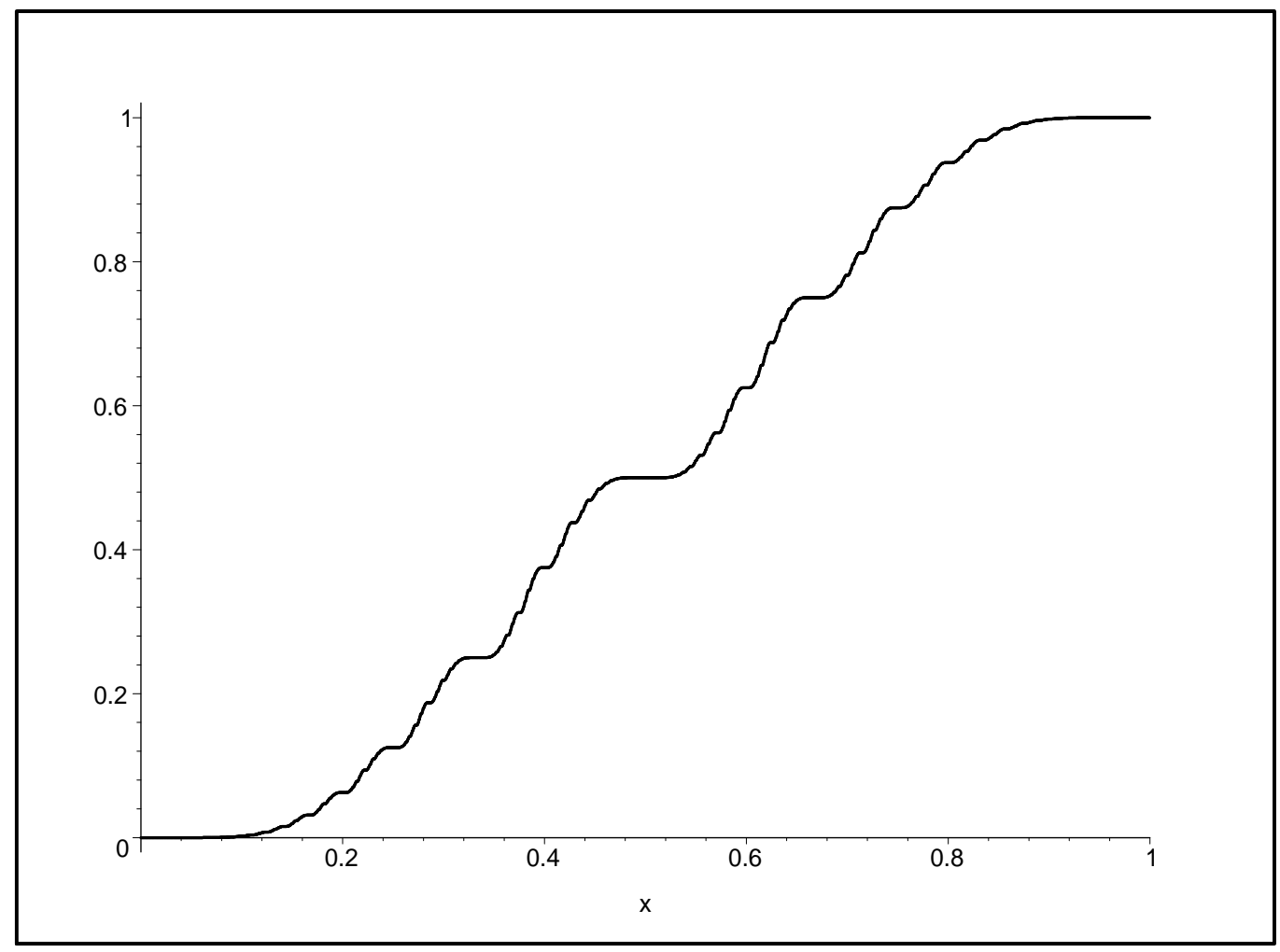

FIGURE 1. Minkowski's question mark $?(x), x \in[0,1]$

Elementary considerations show that this tree contains any positive rational number once and only once in lowest terms [4]. First four iterations lead to

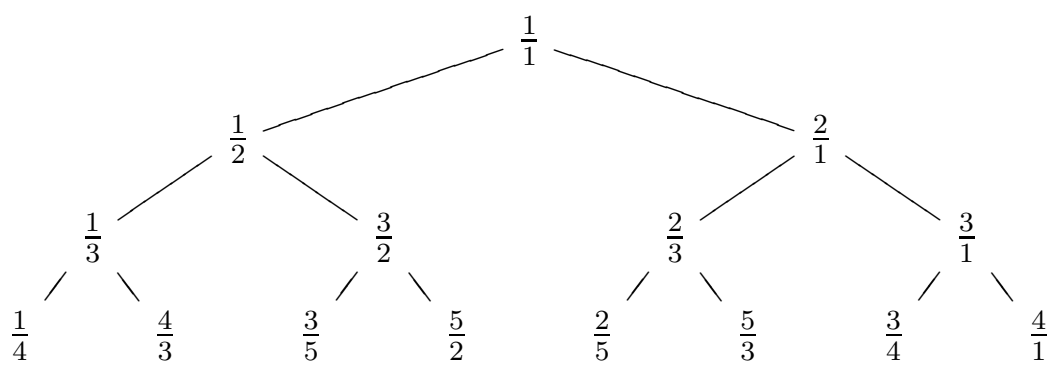

It is important to note that the $n$th generation of the Calkin-Wilf binary tree consists of exactly those rational numbers whose elements of the continued fraction sum up to $n$. This fact can be easily inherited directly from the definition. First, if rational number $\frac{a}{b}$ is represented as a continued fraction $\left[a_{0}, a_{1}, \ldots, a_{r}\right]$, then the map $\frac{a}{b} \rightarrow \frac{a+b}{b}$ maps $\frac{a}{b}$ to $\left[a_{0}+1, a_{1} \ldots, a_{r}\right]$. Second, the map $\frac{a}{b} \rightarrow \frac{a}{a+b}$ maps $\frac{a}{b}$ to $\left[0, a_{1}+1, \ldots, a_{r}\right]$ in case $\frac{a}{b}<1$, and to $\left[1, a_{0}, a_{1}, \ldots, a_{r}\right]$ in case $\frac{a}{b}>1$. This is an important fact which makes the investigations of rational numbers according to their position in the Calkin-Wilf tree highly motivated from the perspective of metric number theory and dynamics of continued fractions. We refer the reader to papers [1], [2] and [3] for a wider overview of the properties of ? $(x)$, 
and to a web page [10] for an exhaustive bibliography list.

The work [1] by the author initiated study of the moments of the Minkowski question mark function. Surprisingly, the only work where an analogous sequence of moments was considered before is [6], where the authors consider the moments of a related singular function $F_{2}(x)$. The moments of the question mark function are defined by

$$
m_{L}=\int_{0}^{\infty}\left(\frac{x}{x+1}\right)^{L} \mathrm{~d} F(x)=2 \int_{0}^{1} x^{L} \mathrm{~d} F(x)=\int_{0}^{1} x^{L} \mathrm{~d} ?(x) .
$$

This sequence is of definite number-theoretical significance because

$$
m_{L}=\lim _{n \rightarrow \infty} 2^{2-n} \sum_{a_{1}+a_{2}+\ldots+a_{s}=n}\left[0, a_{1}, a_{2}, \ldots, a_{s}\right]^{L}
$$

(the summation takes place over rational numbers presented as continued fractions; thus, $s \geq 1, a_{i} \geq 1$ and $\left.a_{s} \geq 2\right)$. As an example of structural properties of the sequence $m_{L}$ we mention the following result. Let $\mathfrak{m}(t)=\sum_{L=0}^{\infty} \frac{m_{L} t^{L}}{L !}$, which is an entire function. One of the main results about $\mathfrak{m}(t)$ is that it is uniquely determined by the regularity condition $\mathfrak{m}(-t) \ll e^{-\sqrt{t \log 2}}$ as $t \rightarrow \infty$, the boundary condition $\mathfrak{m}(0)=1$, and the integral equation [1]

$$
\mathfrak{m}(-s)=\left(2 e^{s}-1\right) \int_{0}^{\infty} \mathfrak{m}^{\prime}(-t) J_{0}(2 \sqrt{s t}) \mathrm{d} t, \quad s \in \mathbb{R}_{+} .
$$

(Here $J_{0}(*)$ stands for the Bessel function $J_{0}(z)=\frac{1}{\pi} \int_{0}^{\pi} \cos (z \sin x) \mathrm{d} x$ ). This reveals the surprising fact that $\mathfrak{m}(t)$ can be considered as the dyadic analogue of entire functions associated with Maass wave forms [9]. Also, the generating function $G(z)=\sum_{L=1}^{\infty} m_{L} z^{L-1}$ can be analytically continued to the cut plane $\mathbb{C} \backslash(1, \infty)$ and it satisfies the three term functional equation

$$
\frac{1}{z}+\frac{1}{z^{2}} G\left(\frac{1}{z}\right)+2 G(z+1)=G(z) .
$$

Minding the specific form of this equation, it is natural to call $G(z)$ the dyadic period function.

It is not clear whether there exists a closed form formula for the moments $m_{L}$. This would be greatly desirable minding the expression (3). On the other hand, one has the following asymptotic result [2]. Let $\mathrm{C}=e^{-2 \sqrt{\log 2}}=0.189169995269+$. Then the following estimate holds, as $L \rightarrow \infty$ :

$$
\mathrm{C}^{\sqrt{L}} \ll m_{L} \ll L^{1 / 4} \mathrm{C}^{\sqrt{L}}
$$


Both implied constants are absolute. The aim of this paper is to find the first exact asymptotic term. Though from our point of view this is of inferior significance than the closed form formula (if the latter does exist), nevertheless, it is of definite interest too.

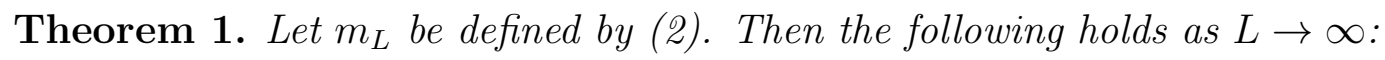

$$
m_{L}=\sqrt[4]{4 \pi^{2} \log 2} \cdot c_{0} \cdot L^{1 / 4} \mathrm{C}^{\sqrt{L}}+O\left(L^{-1 / 4} \mathrm{C}^{\sqrt{L}}\right) .
$$

Here the constant $c_{0}$ is given by $c_{0}=\int_{0}^{1} \Psi(x) \mathrm{d} x=1.030199563382+$, where $\Psi(x)$ is 1 -periodic function given by $\Psi(x)=2^{x}(1-F(x))$ for $x \in[0, \infty)$.

Remark 1. The periodicity of $\Psi(x)$ is obvious from (11). Numerically, $\sqrt[4]{4 \pi^{2} \log 2} \cdot c_{0}=$ $2.356229889908+$. The sequence of moments

$$
M_{L}=\int_{0}^{\infty} x^{L} \mathrm{~d} F(x)
$$

is equally important. These moments represent values of higher left derivatives of $G(z)$ at $z=1$; more precisely, a formal Taylor expansion at $z=1$ is given by

$$
G(z+1)=\sum_{L=1}^{\infty} M_{L} z^{L-1} .
$$

As it was proved in [1], one has the asymptotic formula $M_{L} \sim L ! \frac{c_{0}}{(\log 2)^{L}}$. Moreover, in this case there exists an exact convergent asymptotic series [2]. Though sequences $M_{L}$ and $m_{L}$ are linearly dependent via relations

$$
m_{L}=M_{L}-\sum_{s=0}^{L-1} M_{s}\left(\begin{array}{l}
L \\
s
\end{array}\right), \quad L \geq 0,
$$

it is nevertheless significant that the same structural constant $c_{0}$ does manifest in asymptotic formulae for both of these sequences.

Remark 2. As it is clear from the proof, other terms of asymptotic expansion can be calculated as well. We confine to the first term since calculations are standard (though tedious) with no new ideas being introduced. Note that the structural constants $c_{s}=\int_{0}^{1} x^{s} \Psi(x) \mathrm{d} x$ do appear in the asymptotic expansion, and the method used shares some similarities with the Euler-Maclaurin summation.

Remark 3. If we start from the representation of $m_{L}$ via the last integral of (2) (which is the most natural), then to obtain the representation by the first integral we use the second equation in (11). Moreover, one of the core stages of the proof relies on the periodicity of $\Psi(x)$ and so uses the first equation in (11). Since these two equations are characteristic only to the Minkowski question mark function, generally speaking, our asymptotic formula for the moments of $F(x)$ is unique among similar results for other probabilistic distributions with proper support on the interval $[0,1]$. 


\section{ProOF}

2.1. Preliminary calculations. In this paper we use the notation of Landau to denote by $\mathcal{B}$ some absolutely bounded function in certain neighborhood of a variable; in our case $\mathcal{B}$ depends mostly on $L$ and we consider the case $L \rightarrow \infty$. As a convention, $\mathcal{B}$ stands for different function if considered in another or even the same formula. Occasionally, this notation is used to denote dependence on other variables and in each case it should be clear what variable and neighborhood is implied in a notation $\mathcal{B}$. The main tool of the proof is a Laplace method. The latter is used in finding an asymptotic expansion of certain integrals depending on parameter. Though in our case certain corrections, amendments and variations are necessary, the main technique is standard and can be found, for example, in [8]. Since $1-F(x)=2^{-x} \Psi(x)$, identity (2) implies

$$
\begin{aligned}
m_{L} & =\int_{0}^{\infty}\left(\frac{x}{x+1}\right)^{L} \mathrm{~d}(F(x)-1)=\left.(F(x)-1)\left(\frac{x}{x+1}\right)^{L}\right|_{0} ^{\infty} \\
& +L \int_{0}^{\infty} 2^{-x} \frac{x^{L-1}}{(x+1)^{L+1}} \Psi(x) \mathrm{d} x=L \int_{0}^{\infty} 2^{-x} \frac{x^{L-1}}{(x+1)^{L+1}} \Psi(x) \mathrm{d} x .
\end{aligned}
$$

Put $c=\log 2$. The function $\mathbf{f}(x)=L \cdot \log \frac{x}{x+1}-c x$ achieves its maximum at $x=x_{0}$, where

$$
x_{0}\left(x_{0}+1\right)=\frac{L}{c} ; \text { thus, } x_{0}=\frac{-1+\sqrt{1+\frac{4 L}{c}}}{2}, \text { consequently } x_{0}=\sqrt{\frac{L}{c}}-\frac{1}{2}+\frac{\mathcal{B}}{\sqrt{L}} .
$$

Let $c_{0}=\int_{0}^{1} \Psi(x) \mathrm{d} x$, and let us rewrite the expression for $m_{L}$ as

$$
m_{L}=L c_{0} \int_{0}^{\infty} \frac{1}{x(x+1)} e^{\mathbf{f}(x)} \mathrm{d} x+L \int_{0}^{\infty}\left(\Psi(x)-c_{0}\right) \frac{1}{x(x+1)} e^{\mathbf{f}(x)} \mathrm{d} x=c_{0} L g_{L}+L r_{L} .
$$

Our specific choice in extracting $c_{0}$ out of $\Psi(x)$ as a dominant ingredient can be motivated for the following reason. Since $0.9<\Psi(x)<1.2$, the main weight of the integral defining $m_{L}$ (as far as $\Psi(x)$ is concerned) befalls on a certain constant in the range $(0.9,1.2$ ). Moreover, it is easy to verify that for any continuous 1 -periodic function $\Upsilon(x)$ one has

$$
\begin{aligned}
\int_{-\infty}^{\infty} \Upsilon(x) e^{-A x^{2}} \mathrm{~d} x & \left.=\frac{\sqrt{\pi} \Upsilon(0)}{\sqrt{A}}+O\left(A^{-1}\right) \text { as } A \rightarrow \infty \text { (if } \Upsilon(x) \text { is smooth }\right), \\
\int_{-\infty}^{\infty} \Upsilon(x) e^{-A x^{2}} \mathrm{~d} x & =\frac{\sqrt{\pi} \int_{0}^{1} \Upsilon(x) \mathrm{d} x}{\sqrt{A}}+O(1) \text { as } A \rightarrow 0+
\end{aligned}
$$

This can be seen empirically from the fact that in the first case the dominant weight of the integral is supported only in the neighborhood of 0 , while in the second case this 
interval has a length tending to infinity. As it is seen from the next subsection, we have the second case (a posteriori, the weight of $m_{L}$ is properly supported on the interval $\left(\sqrt{L / c}-L^{2 / 7}, \sqrt{L / c}+L^{2 / 7}\right)$, and hence the correct constant is $c_{0}$ rather then $\Psi\left(x_{0}\right)$.

2.2. Evaluation of $g_{L}$. Choose $\delta=\delta(L)$ such that $\frac{\delta}{L^{1 / 4}} \rightarrow \infty$ and $\frac{\delta}{L^{1 / 3}} \rightarrow 0$. For example, henceforth we fix $\delta=\delta(L)=L^{2 / 7}$. Separate the integral defining $g_{L}$ into four parts:

$$
g_{L}=\int_{0}^{1}+\int_{1}^{x_{0}-\delta}+\int_{x_{0}-\delta}^{x_{0}+\delta}+\int_{x_{0}+\delta}^{\infty} \frac{1}{x(x+1)} e^{\mathbf{f}(x)} \mathrm{d} x=\mathscr{I}_{1}+\mathscr{I}_{2}+\mathscr{I}_{3}+\mathscr{I}_{4} .
$$

2.2.1. Evaluation of $\mathscr{I}_{3}$. First, $\mathbf{f}^{\prime}\left(x_{0}\right)=0$ and $\mathbf{f}^{\prime \prime}\left(x_{0}\right)<0$. Suppose $\left|x-x_{0}\right| \leq \delta$. Then the Taylor formula gives that for certain $\theta_{x} \in\left[x_{0}, x\right]$ one has

$$
\mathbf{f}(x)=\mathbf{f}\left(x_{0}\right)-\alpha\left(x-x_{0}\right)^{2}+\beta\left(x-x_{0}\right)^{3}+\frac{f^{(4)}\left(\theta_{x}\right)}{24}\left(x-x_{0}\right)^{4} .
$$

Direct calculations show that

$$
\begin{aligned}
\mathbf{f}^{\prime \prime}(x) & =-\frac{L(2 x+1)}{x^{2}(x+1)^{2}} ; \text { thus, } \mathbf{f}^{\prime \prime}\left(x_{0}\right)=-\frac{c^{2}}{L}\left(2 x_{0}+1\right) ; \text { this implies } \alpha=\frac{c^{3 / 2}}{\sqrt{L}}+\frac{\mathcal{B}}{L^{3 / 2}} \\
\beta & =\frac{\mathbf{f}^{\prime \prime \prime}\left(x_{0}\right)}{6}=\frac{L\left(3 x_{0}^{2}+3 x_{0}+1\right)}{3 x_{0}^{3}\left(x_{0}+1\right)^{3}}=\frac{c^{2}}{L}+\frac{\mathcal{B}}{L^{3 / 2}} \\
\mathbf{f}^{(4)}(x) & =6 L\left(\frac{1}{(x+1)^{4}}-\frac{1}{x^{4}}\right) ; \text { this gives } \mathbf{f}^{(4)}\left(\theta_{x}\right)=\frac{\mathcal{B}}{L^{3 / 2}} .
\end{aligned}
$$

In the same fashion,

$$
\frac{1}{x(x+1)}=\frac{1}{x_{0}\left(x_{0}+1\right)}+\gamma\left(x-x_{0}\right)+\sigma_{x}\left(x-x_{0}\right)^{2},
$$

where

$$
\gamma=-\frac{2 x_{0}+1}{x_{0}^{2}\left(x_{0}+1\right)^{2}}=-\frac{2 c^{3 / 2}}{L^{3 / 2}}+\frac{\mathcal{B}}{L^{5 / 2}}, \quad \sigma_{x}=\frac{\mathcal{B}}{L^{2}} .
$$

Now let us evaluate the value of $e^{\mathbf{f}\left(x_{0}\right)}$. For $x \rightarrow \infty$, we have

$$
\begin{aligned}
\log \frac{x}{x+1} & =-\frac{1}{x}+\frac{1}{2 x^{2}}+\frac{\mathcal{B}}{x^{3}} ; \text { this gives } \\
L \log \frac{x_{0}}{x_{0}+1} & =-\frac{L}{\sqrt{\frac{L}{c}}-\frac{1}{2}+\frac{\mathcal{B}}{\sqrt{L}}}+\frac{c}{2}+\frac{\mathcal{B}}{\sqrt{L}}=-\sqrt{c L}+\frac{\mathcal{B}}{\sqrt{L}} .
\end{aligned}
$$

Further,

$$
\begin{aligned}
-c x_{0} & =-\sqrt{c L}+\frac{c}{2}+\frac{\mathcal{B}}{\sqrt{L}} ; \text { this gives } \\
e^{\mathbf{f}\left(x_{0}\right)} & =\exp \left(-2 \sqrt{c L}+\frac{c}{2}+\frac{\mathcal{B}}{\sqrt{L}}\right)=e^{-2 \sqrt{c L}} \sqrt{2}\left(1+\frac{\mathcal{B}}{\sqrt{L}}\right) .
\end{aligned}
$$


We already obtained all necessary components to evaluate $\mathscr{I}_{3}$. Note that

$$
\beta\left(x-x_{0}\right)^{3}=\frac{\mathcal{B}}{L} \cdot L^{6 / 7}=o(1), \quad \frac{\mathcal{B}}{L^{3 / 2}}\left(x-x_{0}\right)^{4}=o(1) .
$$

Since $e^{y}=1+y+\mathcal{B} y^{2}$ for $y=o(1)$, the function under the integral (44) in the range $\left(x_{0}-\delta, x_{0}+\delta\right)$ can be written as

$$
\begin{aligned}
\frac{1}{x(x+1)} e^{\mathbf{f}(x)} & =\left(\frac{1}{x_{0}\left(x_{0}+1\right)}+\gamma\left(x-x_{0}\right)+\frac{\mathcal{B}}{L^{2}}\left(x-x_{0}\right)^{2}\right) \\
& \times \exp \left(\mathbf{f}\left(x_{0}\right)-\alpha\left(x-x_{0}\right)^{2}+\beta\left(x-x_{0}\right)^{3}+\frac{\mathcal{B}}{L^{3 / 2}}\left(x-x_{0}\right)^{4}\right) \\
& =e^{\mathbf{f}\left(x_{0}\right)} e^{-\alpha\left(x-x_{0}\right)^{2}} \cdot\left(1+\beta\left(x-x_{0}\right)^{3}+\frac{\mathcal{B}}{L^{3 / 2}}\left(x-x_{0}\right)^{4}+\frac{\mathcal{B}}{L^{2}}\left(x-x_{0}\right)^{6}\right) \\
& \times\left(\frac{1}{x_{0}\left(x_{0}+1\right)}+\gamma\left(x-x_{0}\right)+\frac{\mathcal{B}}{L^{2}}\left(x-x_{0}\right)^{2}\right)
\end{aligned}
$$

(The bound $\left|x-x_{0}\right|=\mathcal{B} L^{1 / 2}$ was used in merging $\frac{\mathcal{B} \beta}{L^{3 / 2}}\left(x-x_{0}\right)^{7}$ and $\frac{\mathcal{B}}{L^{3}}\left(x-x_{0}\right)^{8}$ into $\left.\frac{\mathcal{B}}{L^{2}}\left(x-x_{0}\right)^{6}\right)$. Thus, we have decomposed the function under integral (44) into the sum of twelve functions. It is important to note that two of these functions

$$
e^{\mathbf{f}\left(x_{0}\right)} e^{-\alpha\left(x-x_{0}\right)^{2}} \gamma\left(x-x_{0}\right) \text { and } e^{\mathbf{f}\left(x_{0}\right)} e^{-\alpha\left(x-x_{0}\right)^{2}} \frac{1}{x_{0}\left(x_{0}+1\right)} \beta\left(x-x_{0}\right)^{3},
$$

though being comparatively large, are odd function in $\left(x-x_{0}\right)$ and consequently contribute 0 after integration. We are left with evaluating all the rest summands. This is a routine job. For example,

$$
\begin{aligned}
& e^{\mathbf{f}\left(x_{0}\right)} \gamma \beta \int_{x_{0}-\delta}^{x_{0}+\delta} e^{-\alpha\left(x-x_{0}\right)^{2}}\left(x-x_{0}\right)^{4} \mathrm{~d} x \\
= & e^{\mathbf{f}\left(x_{0}\right)} \gamma \beta \frac{1}{\alpha^{5 / 2}} \int_{-\sqrt{\alpha} \delta}^{\sqrt{\alpha} \delta} y^{4} e^{-y^{2}} \mathrm{~d} y=\mathcal{B} \frac{L^{5 / 4}}{L^{5 / 2}} \mathrm{C}^{\sqrt{L}}=\mathcal{B} L^{-5 / 4} C^{\sqrt{L}} .
\end{aligned}
$$

The second example:

$$
\begin{aligned}
& e^{\mathbf{f}\left(x_{0}\right)} \frac{\mathcal{B} \gamma}{L^{3 / 2}} \int_{x_{0}-\delta}^{x_{0}+\delta} e^{-\alpha\left(x-x_{0}\right)^{2}}\left|x-x_{0}\right|^{5} \mathrm{~d} x \\
= & e^{\mathbf{f}\left(x_{0}\right)} \frac{\mathcal{B} \gamma}{L^{3 / 2} \alpha^{3}} \int_{-\sqrt{\alpha} \delta}^{\sqrt{\alpha} \delta}|y|^{5} e^{-y^{2}} \mathrm{~d} y=\mathcal{B} L^{-3 / 2-3 / 2+3 / 2} \mathrm{C}^{\sqrt{L}}=\mathcal{B} L^{-3 / 2} \mathrm{C}^{\sqrt{L}} .
\end{aligned}
$$

Another example:

$$
e^{\mathbf{f}\left(x_{0}\right)} \frac{\mathcal{B}}{x_{0}\left(x_{0}+1\right) L^{3 / 2}} \int_{x_{0}-\delta}^{x_{0}+\delta} e^{-\alpha\left(x-x_{0}\right)^{2}}\left(x-x_{0}\right)^{4} \mathrm{~d} x=\mathcal{B} \frac{L^{5 / 4}}{L^{5 / 2}} \mathrm{C}^{\sqrt{L}}=\mathcal{B} L^{-5 / 4} \mathrm{C}^{\sqrt{L}} .
$$


Therefore, a direct inspection shows that all functions apart from the main term contribute at most $\mathcal{B} C^{\sqrt{L}} L^{-5 / 4}$ into the value of $g_{L}$. We are left with determining the magnitude of the main term. Since $\sqrt{\alpha} \delta \sim c^{3 / 4} L^{1 / 28}$, this yields

$$
\int_{-\sqrt{\alpha} \delta}^{\sqrt{\alpha} \delta} e^{-y^{2}} \mathrm{~d} y=\int_{-\infty}^{\infty} e^{-y^{2}} \mathrm{~d} y+\mathcal{B} \int_{\sqrt{\alpha} \delta}^{\infty} e^{-y^{2}} \mathrm{~d} y=\sqrt{\pi}+\mathcal{B} \exp \left(-c^{3 / 2} L^{1 / 14}\right) .
$$

And so, the main term is given by

$$
\begin{aligned}
\frac{1}{x_{0}\left(x_{0}+1\right)} e^{\mathbf{f}\left(x_{0}\right)} \int_{-\delta}^{\delta} e^{-\alpha x^{2}} \mathrm{~d} x & =\left(\sqrt{\pi}+\mathcal{B} \exp \left(-c^{3 / 2} L^{1 / 14}\right)\right) \frac{1}{\sqrt{\alpha}} \frac{1}{x_{0}\left(x_{0}+1\right)} e^{\mathbf{f}\left(x_{0}\right)} \\
& =\frac{c}{L} \sqrt{2 \pi} e^{-2 \sqrt{c L}} \sqrt[4]{L} c^{-3 / 4}\left(1+\frac{\mathcal{B}}{\sqrt{L}}\right) .
\end{aligned}
$$

Thus, we obtain the main asymptotic term

$$
\mathscr{I}_{3}=\sqrt[4]{4 \pi^{2} \log 2} \cdot L^{-3 / 4} C^{\sqrt{L}}+\mathcal{B} L^{-5 / 4} C^{\sqrt{L}} .
$$

2.2.2. Evaluation of $\mathscr{I}_{1}, \mathscr{I}_{2}$ and $\mathscr{I}_{4}$. Trivially,

$$
\mathscr{I}_{1}=\int_{0}^{1} \frac{x^{L-1}}{(x+1)^{L+1}} 2^{-x} \mathrm{~d} x=\mathcal{B} 2^{-L} .
$$

To evaluate $\mathscr{I}_{2}$, we use a standard inequality

$$
\log \left(\frac{x}{x+1}\right)<-\frac{1}{x}+\frac{1}{2 x^{2}} \text { for } x \geq 1 .
$$

Thus, since $\mathbf{f}(x)$ is an increasing function in the interval $\left[1, x_{0}\right]$, we have

$$
\mathscr{I}_{2}=\int_{1}^{x_{0}-\delta} \frac{1}{x(x+1)} e^{\mathbf{f}(x)} \mathrm{d} x<\log 2 \cdot e^{\mathbf{f}\left(x_{0}-\delta\right)}=\mathcal{B} e^{\mathbf{f}\left(x_{0}-\delta\right)} .
$$

Further, using the inequality (5), we have

$$
\begin{aligned}
\mathbf{f}\left(x_{0}-\delta\right) & =L \log \left(\frac{x_{0}-\delta}{x_{0}-\delta+1}\right)-c\left(x_{0}-\delta\right)<-\frac{L}{x_{0}-\delta}+\frac{L}{2\left(x_{0}-\delta\right)^{2}}-c x_{0}+c \delta \\
& =-\frac{L}{x_{0}}-c x_{0}+\mathcal{B}+c \delta+\left(\frac{L}{x_{0}}-\frac{L}{x_{0}-\delta}\right) \\
& =-\frac{L}{x_{0}}-c x_{0}+\mathcal{B}+c \delta-\frac{L \delta}{x_{0}\left(x_{0}+1\right)}-L \delta\left(\frac{1}{x_{0}\left(x_{0}-\delta\right)}-\frac{1}{x_{0}\left(x_{0}+1\right)}\right) \\
& =-\frac{L}{x_{0}}-c x_{0}+\mathcal{B}-\frac{L \delta^{2}}{x_{0}^{3}}=-2 \sqrt{c L}+\mathcal{B}-c^{3 / 2} L^{1 / 14}
\end{aligned}
$$

(recall that $\delta=L^{2 / 7}$ ). Therefore,

$$
\mathscr{I}_{2}=\mathcal{B} C^{\sqrt{L}} \exp \left(-c^{3 / 2} L^{1 / 14}\right)=\mathcal{B} L^{-5 / 4} C^{\sqrt{L}} .
$$


In the same vein,

$$
\mathbf{f}\left(x_{0}+\delta\right)=-2 \sqrt{c L}+\mathcal{B}-c^{3 / 2} L^{1 / 14}
$$

Since $\mathbf{f}(x)$ is decreasing in the interval $\left[x_{0}, \infty\right)$, we obtain

$$
\begin{aligned}
\mathscr{I}_{4} & =\int_{x_{0}+\delta}^{\infty} \frac{1}{x(x+1)} e^{\mathbf{f}(x)} \mathrm{d} x<e^{\mathbf{f}\left(x_{0}+\delta\right)} \int_{1}^{\infty} \frac{\mathrm{d} x}{x(x+1)} \\
& =\mathcal{B} C^{\sqrt{L}} \exp \left(-c^{3 / 2} L^{1 / 14}\right)=\mathcal{B} L^{-5 / 4} C^{\sqrt{L}} .
\end{aligned}
$$

Combining all the estimates for $\mathscr{I}_{1}, \mathscr{I}_{2}, \mathscr{I}_{3}$ and $\mathscr{I}_{4}$, we eventually obtain

$$
g_{L}=\sqrt[4]{4 \pi^{2} \log 2} \cdot L^{-3 / 4} \mathrm{C}^{\sqrt{L}}+\mathcal{B} L^{-5 / 4} \mathrm{C}^{\sqrt{L}}
$$

2.3. Evaluation of $r_{L}$. In this subsection we can be more concise, since the method is the same as we used to evaluate $g_{L}$. Recall that

$$
r_{L}=\int_{0}^{\infty}\left(\Psi(x)-c_{0}\right) \frac{1}{x(x+1)} e^{\mathbf{f}(x)} \mathrm{d} x
$$

Let

$$
\hat{\Psi}(x)=\int_{0}^{x}\left(\Psi(t)-c_{0}\right) \mathrm{d} t .
$$

The definition of $c_{0}$ yields that $\hat{\Psi}(x)$ is bounded (and periodic) function. Using an integration by parts, we can rewrite an expression for $r_{L}$ as

$$
r_{L}=\int_{0}^{\infty} \hat{\Psi}(x) h(x) e^{\mathbf{f}(x)} \mathrm{d} x ; \text { thus, } r_{L}=\mathcal{B} \int_{0}^{\infty} h(x) e^{\mathbf{f}(x)} \mathrm{d} x
$$

where

$$
h(x)=\frac{1}{x^{2}}-\frac{1}{(x+1)^{2}}-\frac{1}{x(x+1)} \mathbf{f}^{\prime}(x)=-\frac{L}{x^{2}(x+1)^{2}}+\frac{c}{x(x+1)}+\frac{2 x+1}{x^{2}(x+1)^{2}} .
$$

Note that

$$
h\left(x_{0}\right)=\frac{2 x_{0}+1}{x_{0}^{2}\left(x_{0}+1\right)^{2}}=\frac{2 c^{3 / 2}}{L^{3 / 2}}+\frac{\mathcal{B}}{L^{5 / 2}} .
$$

As a matter of fact, we have a completely analogous integral to the one defining $g_{L}$, with the major difference in the estimate $h\left(x_{0}\right)=\mathcal{B} L^{-3 / 2}$, whereas $\frac{1}{x_{0}\left(x_{0}+1\right)}=\mathcal{B} L^{-1}$. Thus, using the same method to evaluate $r_{L}$ as we did with $g_{L}$, one gets

$$
r_{L}=\mathcal{B} L^{1 / 4-3 / 2} \mathrm{C}^{\sqrt{L}}=\mathcal{B} L^{-5 / 4} \mathrm{C}^{\sqrt{L}} .
$$

Since $m_{L}=c_{0} L g_{L}+L r_{L}$, this finishes the proof of Theorem 1 . 
2.4. Final remarks. As can be inherited from the proof, the share of integrals $\mathscr{I}_{1}, \mathscr{I}_{2}$ and $\mathscr{I}_{4}$ into the exact value of $m_{L}$ is of order $C^{\sqrt{L}} \exp \left(-c^{3 / 2} L^{1 / 14}\right)$. Thus, a standard machinery of an asymptotic expansion shows that all terms for asymptotic series of $m_{L}$ are supported on the neighborhood $\left(x_{0}-\delta, x_{0}+\delta\right)$. More thorough inspection reveals that

$$
m_{L} \sim L^{1 / 4} C^{\sqrt{L}} \cdot \sum_{i=0}^{\infty} \frac{\mathrm{A}_{i}}{L^{i / 2}}
$$

As was mentioned, every term of this expansion can be obtained by (increasingly tedious) computations. For example, $\mathrm{A}_{1}$ is a sum of $c_{0}$ and $c_{1}=\int_{0}^{1} x \Psi(x) \mathrm{d} x$, each multiplied by some explicit constant (constant $c_{1}$ occurs while extracting the main term of the integral $r_{L}$ ). Nevertheless, from a number-theoretic point of view our main concern is the structure of values $m_{L}$ rather than their magnitude (though dominant) in some small neighborhood of $x_{0}$, and hence we have confined only in exhibiting such possibility of asymptotic expansion.

We finish with providing a table for some values of constants $m_{L}$.

\begin{tabular}{|r|l|l|}
\hline \multicolumn{3}{|c|}{ Sequence $m_{L}$} \\
\hline$L$ & $m_{L}$ & $m_{L}^{\star}$ \\
\hline 1 & 0.5000000000 & 2.643125297 \\
2 & 0.2909264764 & 2.577573745 \\
3 & 0.1863897146 & 2.533204605 \\
4 & 0.1269922584 & 2.509329792 \\
5 & 0.09016445494 & 2.496320715 \\
6 & 0.06592816257 & 2.488147649 \\
7 & 0.04929431046 & 2.481940613 \\
8 & 0.03751871185 & 2.476544438 \\
9 & 0.02897962203 & 2.471583746 \\
10 & 0.02266585817 & 2.466982861 \\
11 & 0.01792085923 & 2.462750421 \\
12 & 0.01430468951 & 2.458897371 \\
20 & 0.003008686707 & 2.438565967 \\
30 & 0.0006211064464 & 2.425096683 \\
40 & 0.0001622371309 & 2.416702495 \\
50 & 0.00004937221843 & 2.410831724 \\
100 & 0.0000004445933003 & 2.395743861 \\
\hline
\end{tabular}

Here $m_{L}^{\star}=\frac{m_{L}}{\sqrt[4]{L} \mathrm{C} \sqrt{L}}$. The main result of the paper implies that the sequence $m_{L}^{\star}$ tends to the limit $c_{0} \sqrt[4]{4 \pi^{2} \log 2}=2.3562298899+$. Unfortunately, we do not have yet any evidence that the closed form expression exists for $c_{0}$ (as well as for $m_{L}$ with $L \geq 2$ ). Finally, 
we remark that the result of Theorem 1 should be considered in conjunction with linear relations [1] $m_{L}=\sum_{s=0}^{L}\left(\begin{array}{c}L \\ s\end{array}\right)(-1)^{s} m_{s}, L \geq 0$, which the sequence $m_{L}$ satisfies.

\section{REFERENCES}

[1] G. Alkauskas, The moments of Minkowski question mark function: the dyadic period function (submitted); arXiv:0801.0051.

[2] G. Alkauskas, Generating and zeta functions, structure, spectral and analytic properties of the moments of Minkowski question mark function (submitted); arXiv:0801.0056.

[3] G. Alkauskas, Minkowski question mark function and its generalizations, associated with pcontinued fractions: fractals, explicit series for the dyadic period function and moments (submitted); arXiv:0805.1717.

[4] N. Calkin, H. Wilf, Recounting the rationals, Amer. Math. Monthly, 107:360-363, 2000.

[5] A. Denjoy, Sur une fonction réelle de Minkowski, J. Math. Pures Appl., 17:105-151, 1938.

[6] P.J. Grabner, P. Kirschenhofer, R.F. Tichy, Combinatorial and arithmetical properties of linear numeration systems, Combinatorica, 22(2):245-267, 2002.

[7] A.Ya. Khinchin, Continued fractions, The University of Chicago Press, 1964

[8] M.A. Lavrent'ev, B.V. Shabat, Methods of the theory of functions of a complex variable, Nauka, Moscow, 1987 (in Russian).

[9] J.B. LewIS, Spaces of holomorphic functions equivalent to the even Maass cusp forms, Invent. Math., 127(2):271-306, 1997.

[10] An exhaustive bibliography on the Minkowski question mark function, http://www.maths.nottingham.ac.uk/personal/pmxga2/minkowski.htm

The School of Mathematical Sciences, The University of Nottingham, University Park, Nottingham NG7 2RD United Kingdom

Vilnius University, The Department of Mathematics and Informatics, Naugarduko 24, Vilnius, Lithuania

giedrius.alkauskas@maths. nottingham.ac.uk 